$3^{\text {rd }}$ International Symposium on Integrating CFD and Experiments in Aerodynamics

\title{
Accurate Flow Prediction for Store Separation from Internal Bay
}

\author{
M. Mani ${ }^{1}$, A.W. Cary, ${ }^{2}$ W.W. Bower ${ }^{3}$ \\ 1,2,3, The Boeing Company mori.mani@boeing.com \\ P.O. Box 516, MC-S111-1240 \\ St. Louis Missouri
}

\section{Introduction}

Accurate prediction of store separation from internal aircraft bays is of great importance in commercial and military aircraft design. The flow-field inside and over the bay is very complex, especially for supersonic flows involving shock-boundary layer interaction, compressible shear layer, and strong acoustics. Understanding the physics of the flow in and surrounding the bay is of great importance for safe release of stores and controlling the acoustic environment.

The supersonic flow-field within and surrounding the bay is unsteady, and accurate prediction of the shear layer requires dynamic simulations with advanced numerical and physical models. The objective of this paper is to demonstrate accurate prediction of the effect of the flow and a control method by employing a turbulence model that is not cost-prohibitive and is accurate enough to simulate such complex flow phenomena. The turbulence model employed for this study is a twoequation hybrid Reynolds-Averaged Navier-Stokes (RANS) [1] and Large Eddy Simulation (LES) [2] formulation known as the Large Eddy Stress balanced (LESb) model [3].

\section{Turbulence Model}

The two-equation RANS models use $k$ to represent all of the unsteady fluctuations, while the LES models use $k$ to represent only the spatial average of the fluctuations within a filter width. The length scale $l_{B}$ in the balanced LES model (LESb) is defined as:

$l_{B}=\min \left(l_{\varepsilon}, C_{B} \Delta\right)$

and the turbulent viscosity is represented as:

$\mu_{t}=\bar{\rho} C_{\mu} l_{B} \sqrt{k}$

For unresolved length scales $\left(\Delta>>l_{\varepsilon}\right.$ ) the model reverts to the standard two-equation model. However, for resolved length scales $\left(\Delta<<l_{\varepsilon}\right)$, the model reverts to the LES model, and as the resolved length scale goes to zero (full resolution of all pertinent scales) the model approaches a Direct Numerical Simulation (DNS) of turbulence.

Implementation of the LESb model in an existing RANS code with a two-equation model (and the ability to run time-accurate simulations) is straightforward. The existing model solves for $\omega$, which implies an effective length scale of the turbulence and enters the $k$ equation as a dissipation rate of the turbulent kinetic energy (TKE). To implement the model, one should compare the computed effective length scale with the grid scale, and limit the dissipation term accordingly. Then one can implement the filter as follows:

$\omega_{B}=\max \left(\omega, \frac{k^{1 / 2}}{C_{\mu} C_{B} \Delta}\right)$

The effect of the limiter is to increase the dissipation of turbulent kinetic energy. Thus the TKE is reduced from that predicted by the traditional turbulence model such that 
$3^{\text {rd }}$ International Symposium on Integrating CFD and Experiments in Aerodynamics

20-21 June 2007

U.S. Air Force Academy, CO, USA

length scales that are resolved do not contribute to the Reynolds stress terms.

For isotropic grids, $\Delta$ is defined as

$\Delta=(\text { Volume })^{1 / 3}=(1 / J)^{1 / 3}$

where $J$ is the Jacobian of the grid transformation. For stretched grids, the model assumes that the smallest resolved eddies should be roughly isotropic and so must be resolved in all three coordinate directions, and in time. Thus the filter is defined as:

$\Delta=\max (d x, d y, d z, u * d t, \sqrt{k} * d t)$

The limiting length scales based on time-step represent the scales based on convection velocity and SGS turbulence respectively. These scales are included to ensure that there is sufficient time resolution to resolve the captured physical phenomena. That is, if the time steps are too large, the unsteady phenomena cannot be resolved, and the RANS model should be used.

Near the wall in typical boundary layer grids, $d y$ will be small to resolve the mean shear, but $d x$ is typically very large. Thus the LESb model should revert to the underlying two-equation model near walls. However, as the boundary layer is traversed into grids that are more isotropic and resolve the large-scale structures, the model should smoothly transition to an LES model.

\section{Grid and Solutions}

To understand the impact of the shear layer from an internal bay, we will begin our study with a supersonic flow over an empty cavity; then we will investigate how to actively control the shear layer and suppress resonance in the bay by blowing with a sonic slot-jet upstream of the bay. A similar study was carried out experimentally by Zhuang, et.al [4] and a numerical investigation of approximately similar geometry using LES turbulence model was carried out by Rizzetta, et.al [5].

A 3D grid was generated for a cavity $(\mathrm{L}=12, \mathrm{D}=2.4, \mathrm{~W}=2.1$ centimeters) with an aspect ratio of $\mathrm{L} / \mathrm{D}=5.0$ The geometry contains the upstream plane to capture the boundary layer accurately and side panels to include the boundary layer interaction with the shear layer. The computational grid contains 2.7 million points. To avoid a small time-step in advancing the governing equation in time, the grid spacing at the wall is set to $y^{+}=20$. The wall function [6] is invoked point by point when the $\mathrm{y}^{+}$is greater than 15 . The grid is shown for every other point in Figure 1. The freestream flow conditions are Mach $=2.0, \mathrm{P}_{0}=2.17 \times 10^{5} \mathrm{~Pa}$, and $\mathrm{T}_{0}=336 \mathrm{~K}, \mathrm{Re} / \mathrm{cm}=2.33 \times 10^{5}$. The unsteady cavity solutions were obtained with symmetric and full configurations with and without control to investigate the asymmetric effect of the turbulence model. The plenum total pressure and temperature for the slot-jet are $217.00 \mathrm{psi}$ and $621.6{ }^{0} \mathrm{R}$.

The solutions were obtained using the BCFD code [7]. The BCFD code is an implicit generalpurpose Euler and Navier-Stokes solver with hybrid unstructured and structured/unstructured grids. Numerous special algorithms from first-order to fifth-order accurate are available for structured grids applications.

The Reynolds average Navier-Stokes equations with the hybrid turbulence model of Reference 3 are used to advance the governing equations in time. The Roe scheme with total variation diminishing, which is second-order accurate in the physical domain, was employed in this study. Since the computational domain was split into 26 zones, a global Newton with five sub-iterations was employed to unify the zonal solution in time. A $\Delta \mathrm{t}$ of 1.0 microsecond was employed to advance the solution in time, which will provide one free-stream convective time scale in 200 time-steps. 
$3^{\text {rd }}$ International Symposium on Integrating CFD and Experiments in Aerodynamics

20-21 June 2007

U.S. Air Force Academy, CO, USA

\section{Results}

Two unsteady solutions with the hybrid turbulence model were obtained. The time-mean of pressure contours at the center plane of the cavity is shown in Figure 2. The pressure in and over the cavity clearly has been suppressed by the slot-jet. There is a region of high pressure at the aft wall followed by a low pressure region in the uncontrolled case. The pressure in the same region for the controlled case is lower. The pressure along the cavity wall at the centerline is plotted for both cases in Figure 3. The pressure is lower at all locations for the controlled case. However, the lowest pressure is at $x=12.0$ inches. The time-mean of the stream-wise velocity contours is shown in Figure 4. There is a separation region upstream of the slot-jet which causes a secondary shock that combines with the slot-jet shock and forms a lambda shock. A thickening of the boundary layer can be observed upstream of the lambda shock as a result of this separation.

Instantaneous snapshots of the density gradient without and with control at $t=t_{1}$ and $t_{2}$ are shown in Figures 5 and 6 . The shock structure due to mass injection substantially changes the flow structure in the shear layer and cavity. The shear layer is lifted off the bay, and as a result there is reduced interaction between the shear layer and the aft end of the bay. This would substantially diminish the sonic fatigue by damping the feedback loop generated by the shear layer interacting with the aft face of the cavity. In the controlled case, the cavity leading edge shock is a pulsating shock. This shock is formed due to the entrainment of the flow across the leading edge of the bay. The iso-surfaces of vorticity magnitudes are shown with the density gradient in Figure 7 with and without mass injection at time $t_{2}$. The iso-surface for the uncontrolled flow shows substantially more three-dimensionality in the shear layer than the controlled flow shear layer. Figure 8 shows the density gradient at the center plane with several axial cuts along the cavity for both cases (with and without slot-jet). The interaction of the cavity side on the shear layer flow and the extent that the free shear layer extends beyond the edge into the bay can be seen. In the downstream of the cavity, flow is being sucked into the cavity from the side wall, while in the upstream region, flow is spilling out of the cavity over the side wall, creating a weak vortex.

The snapshot of the Mach, velocities, and span-wise vorticities are shown at two time intervals in Figures 9-12. The dynamic of the cavity flow is formed by the large structures formed in the upstream section of the cavity that convects downstream as the unsteady shear layer oscillates over the cavity.

The pressure spectra at three different locations at mid-span on the upstream, center and aft cavity walls are shown in Figure 13. The coordinate locations are $x=20.3,25.4,32.5 ; y=-0.3,-2.4,-0.3$; $\mathrm{z}=0.0$ centimeters accordingly. The primary cavity tones are seen in the 1000 to $3000 \mathrm{~Hz}$ frequencies, with primary peak at $3000 \mathrm{~Hz}$. There are no significant differences in the magnitude of the frequencies in the upstream and cavity floor. However, the magnitude of the SPL is greater by about $20 \mathrm{~dB}$ on the downstream wall just below the cavity lip. The comparison of the SPL between no flow control and flow-control shows that the primary peaks have been eliminated and the broadband spectrum has been reduced by about 10 to $20 \mathrm{~dB}$ when the control is on. The experimental data of Reference 2 shows about the same order of magnitude reduction in the SPL as we have observed in the CFD study. However, in their cases the pressure peaks are about 20 $\mathrm{dB}$ below what CFD has predicted.

\section{Conclusions}

The hybrid LESb turbulence model is a cost-efficient approach to predicting complex high shear flow-field and massively separated flows. The results indicate that the slot-jet has significant impact $(20 \mathrm{~dB})$ in suppressing the acoustic load and the behavior of the shear layer. 
U.S. Air Force Academy, CO, USA

\section{References}

[1] Menter, F.R., "Zonal Two Equation k- $\omega$ Turbulence Models for Aerodynamic Flows," AIAA Paper Number 93-2906, $24^{\text {th }}$ Fluid Dynamics Conference, July 6-9, 1993, Orlando Florida

[2] Smagorinsky, J., "General Circulation Experiments With the Primitive Equations," Mon. Weather Rev., 91, 99-164.

[3] Bush, R.H., and Mani, M., "A Two-equation LES/RANS Hybrid Turbulence Model for High Sub-grid Shear Model," AIAA 2001-2561.

[4] Zhuang, N., Alvi, F.S., Alkislar, M.B., and Shih, C., "Aeroacoustic Properties of Supersonic Cavity Flows and Their Control," AIAA 2003-3101, $9^{\text {th }}$ AIAA/CEAS Aeroacoustics Conference, May 2003.

[5] Rizzetta, D.P., and Visbal, M.R., "Large-Eddy Simulation of Supersonic Cavity Flowfields Including Flow Control," AIAA Journal, Vol. 41, No. 8, August 2003.

[7] Mani, M., Cary, A.W., and Ramakrishnan, S.V., "A Structured and Hybrid-unstructured Grid Euler and Navier-Stokes Solver for General Geometry," AIAA 2004-524. 
$3^{\text {rd }}$ International Symposium on Integrating CFD and Experiments in Aerodynamics

20-21 June 2007

U.S. Air Force Academy, CO, USA
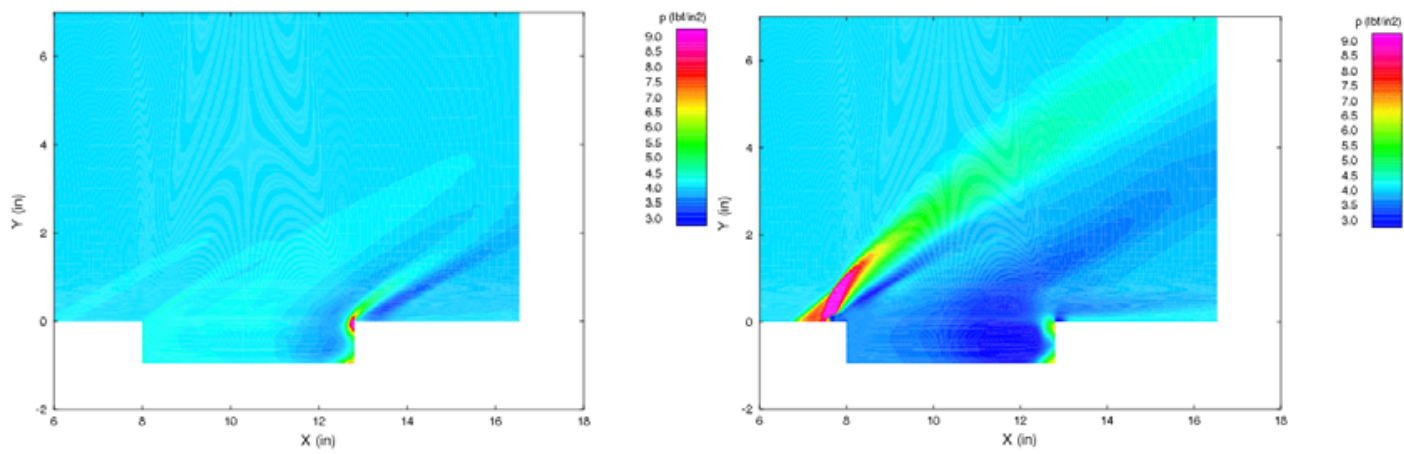

Figure 2 - Pressure contours with and without control at mid-span

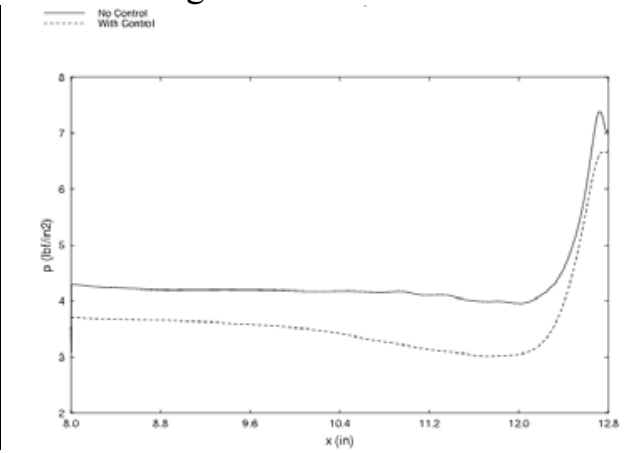

Figure $3-\mathrm{P}_{\mathrm{rms}}$ along the cavity floor

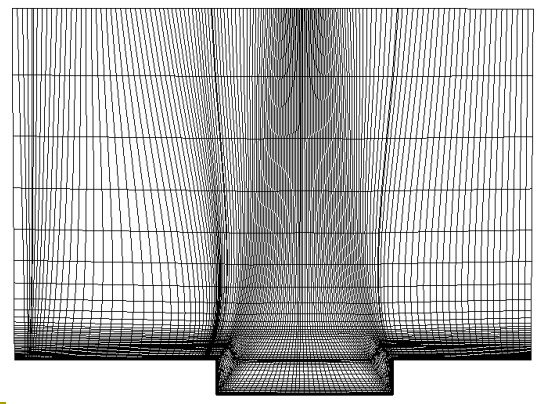

Figure 1 - Center plane grid at every other point
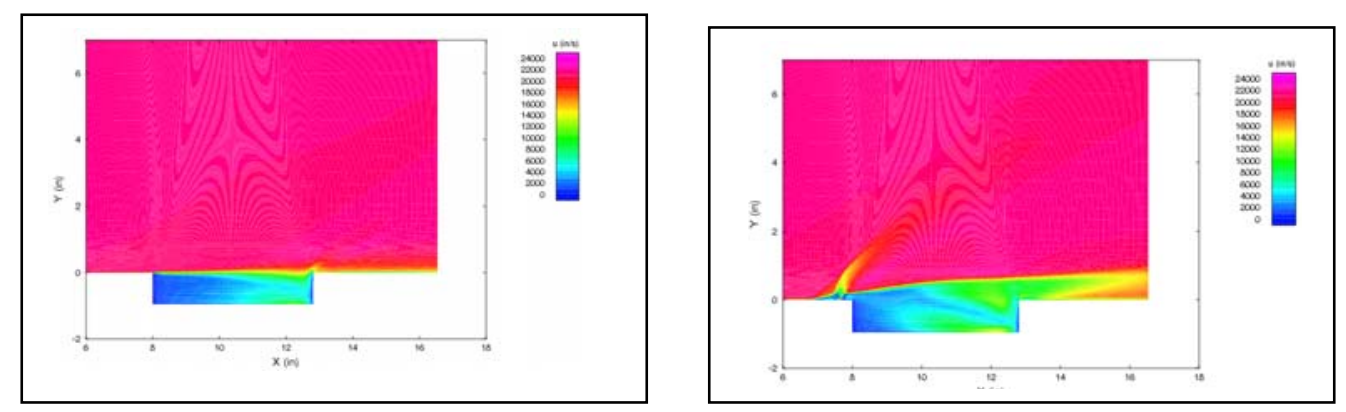

Figure 4 - Time mean of the stream-wise velocities at mid-span 
$3^{\text {rd }}$ International Symposium on Integrating CFD and Experiments in Aerodynamics

20-21 June 2007

U.S. Air Force Academy, CO, USA


Figure 5 - Instantaneous density gradient without slot-jet at time $=\mathrm{t}_{1}, \mathrm{t}_{2}$
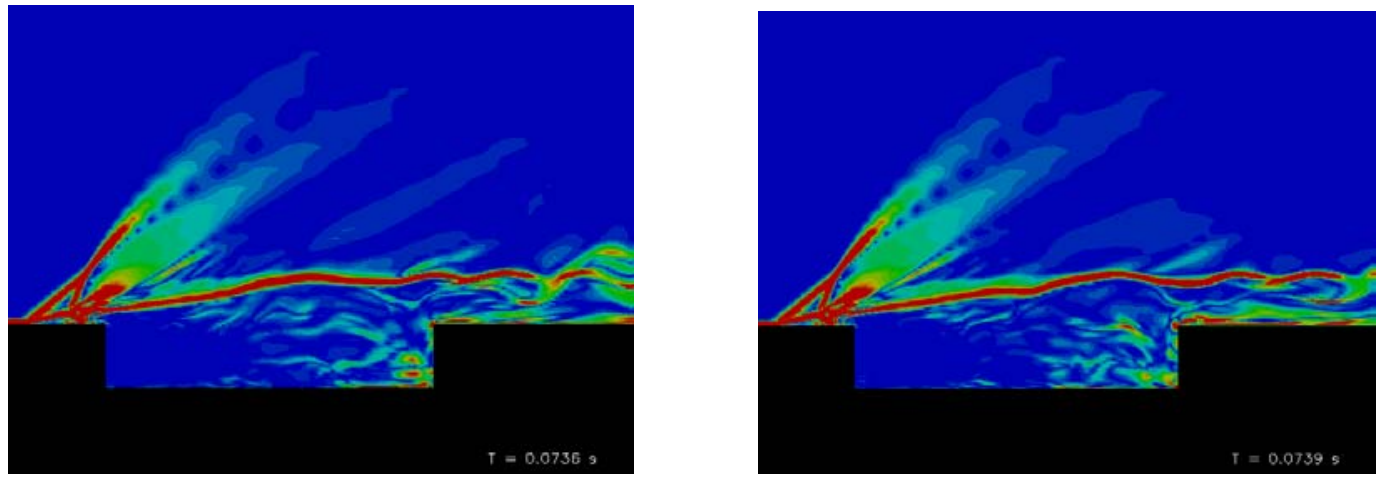

Figure 6 - Instantaneous density gradient with slot-jet on at time $=t_{1}, t_{2}$


Figure 7 - Instantaneous density gradient with iso-surfaces of vorticity magnitude at time $=\mathrm{t}_{2}$
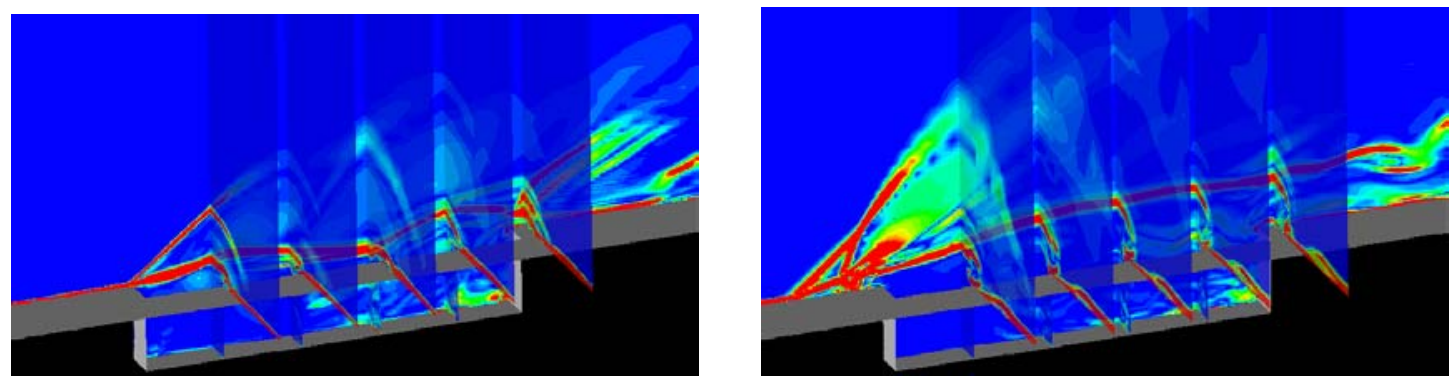

Figure 8 - Uncorrelated instantaneous density gradient at mid-span and several axial flow planes 
$3^{\text {rd }}$ International Symposium on Integrating CFD and Experiments in Aerodynamics

20-21 June 2007

U.S. Air Force Academy, CO, USA
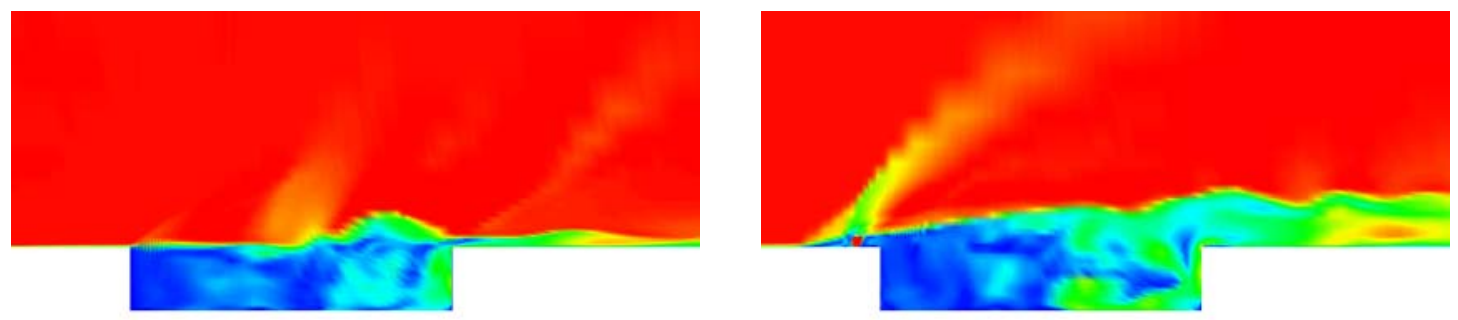

Figure 9 - Instantaneous Mach contours without and with AFC at time $t_{3}$
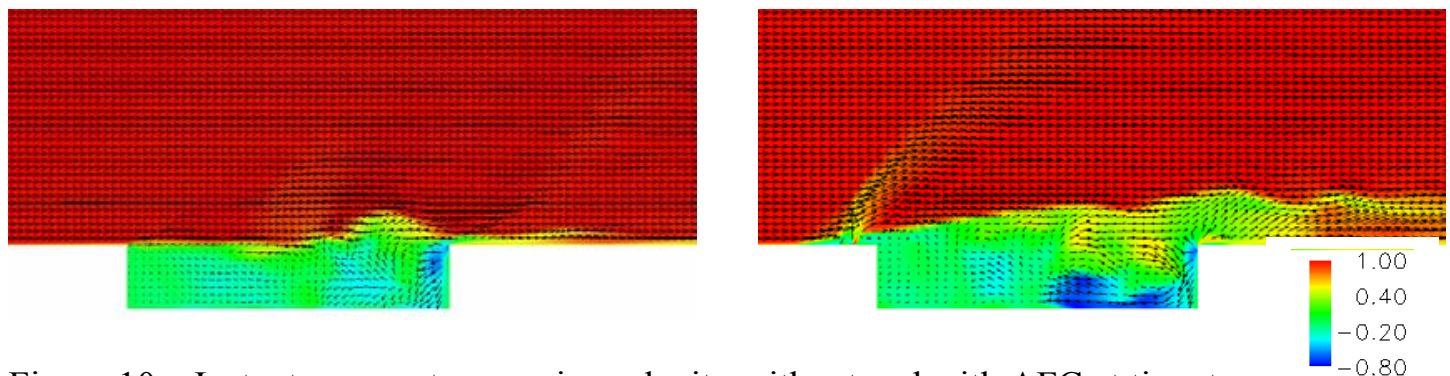

Figure 10 - Instantaneous stream-wise velocity without and with AFC at time $t_{3}$
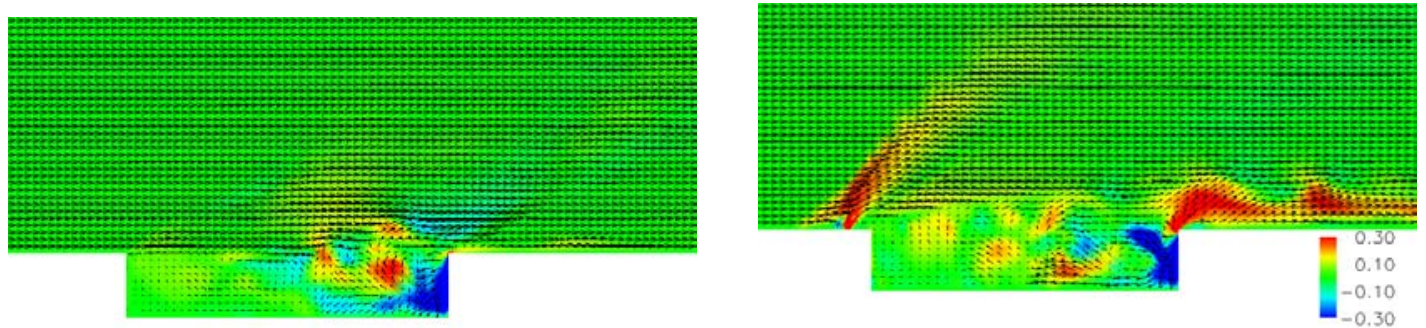

Figure 11 - Instantaneous normal velocity without and with AFC at time $\mathrm{t}_{3}$
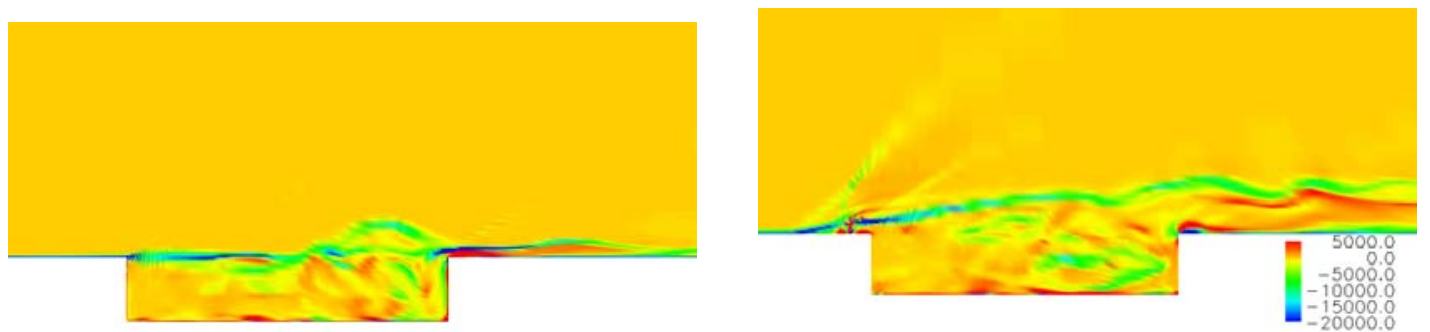

Figure 12 - Instantaneous span-wise vorticities without and with AFC at time $t_{3}$
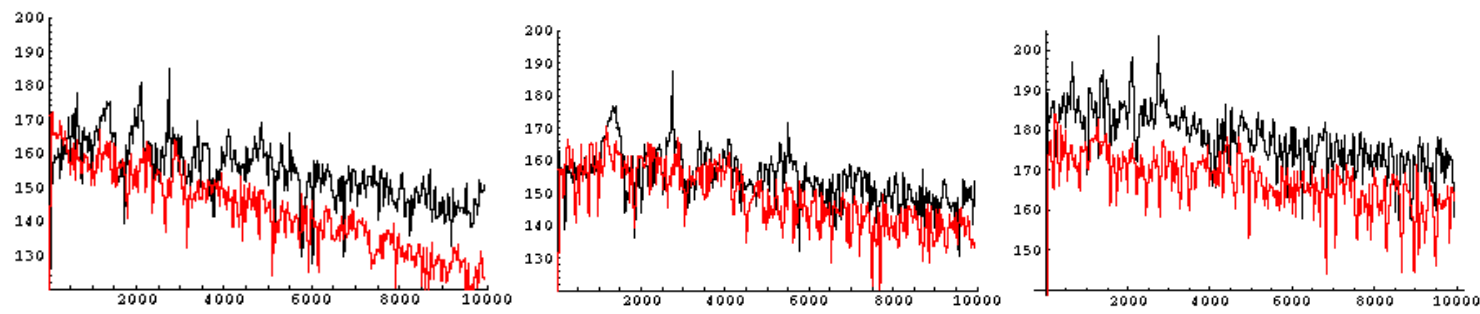

$\mathrm{Hz}$

Figure 13 -Pressure spectra at mid-span on the upstream, bottom center, and aft cavity wall. 